\title{
Cotunneling Transport at Singlet-Triplet Transition in Carbon Nanotube Quantum Dots
}

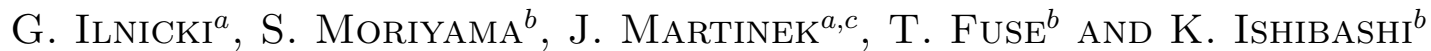 \\ ${ }^{a}$ Institute of Molecular Physics, Polish Academy of Sciences \\ M. Smoluchowskiego 17, 60-179 Poznań. Poland \\ ${ }^{b}$ Advanced Device Laboratory, RIKEN \\ 2-1, Hirosawa, Wako, Saitama 351-0198, Japan \\ ${ }^{c}$ Institute for Materials Research, Tohoku University, Sendai 980-77, Japan
}

\begin{abstract}
Electronic transport through a quantum dot in the Coulomb-blockade cotunelling regime is investigated both experimentally and theoretically, where a single-wall carbon-nanotube weakly coupled to metallic leads plays a role of the dot. We observe a pronounced current peak for the singlet-triplet transition in a half-shell filling regime due to inelastic cotunneling processes. Using the second order perturbation theory we explain physical mechanisms determining the details of signal.
\end{abstract}

PACS numbers: 73.22.-f, 73.23.Hk, 73.63.Fg

\section{Introduction}

Single-walled carbon-nanotubes (SWNTs) are considered as a basic element of future carbon-based electronic systems [1-4]. In this paper we investigate experimentally and theoretically transport properties of a quantum-dot (QD) formed in a SWNT weakly coupled to metallic electrodes in the Coulomb-blockade $(\mathrm{CB})$ regime. In this regime, cotunelling of pairs of electrons dominates the transport [5-8]. We calculate this second order tunneling process exactly using perturbation theory in a nonequilibrium regime $[9,10]$. Comparison with the experimental data serves as a very good test for the theory. We study in detail a singlet-triplet transition occurring in two-orbital SWNT QD in a half-shell filling regime (for two electrons in a dot) [11-14]. A new experimental approach - the detection of the inelastic resonance in respect of magnetic field makes it possible to separate two components of the current (elastic and inelastic) as well as extracting values of various parameters determining the transport. Furthermore, the use of the method enables us to determine the presence of spin-flip and orbital-orbital relaxation processes in SWNT QDs.

\section{Model}

We model our SWNT QD with two orbitals, which gives four possible quantum states marked with orbital number $m=A, B$ and with spin sign $\sigma$. Energy difference between spin states $\epsilon_{m \uparrow}-\epsilon_{m \downarrow}=\Delta_{\mathrm{Z}}=g \mu_{\mathrm{B}} B$ is induced by a magnetic field $B$, and orbital energy difference is given by $\delta=\epsilon_{B \sigma}-\epsilon_{A \sigma}$. The left and right electrodes are marked with $r=\mathrm{L}, \mathrm{R}$ and lead electron momentum states with $k$. An important factor is tunnel coupling strength $\Gamma_{r}^{\alpha}=2 \pi \rho_{r \sigma}\left|V_{r m}\right|^{2}$ (where $V_{r m}$ are tunneling amplitudes; we neglect the spin dependence: $\left.V_{r m k}=V_{r m}\right)$, which may differ for both orbitals leading to an effective pseudospin asymmetry, even though we assume the full spin density of states symmetry $\left(\rho_{r \uparrow}=\rho_{r \downarrow}\right)$ in the leads (as if they were nonmagnetic). Finally, the full form of the Hamiltonian is given by

$$
\begin{aligned}
H= & \sum_{r k \sigma} \varepsilon_{r k \sigma} c_{r k \sigma}^{\dagger} c_{r k \sigma}+\epsilon_{m \sigma} \sum_{m \sigma} d_{m \sigma}^{\dagger} d_{m \sigma} \\
& +\frac{U}{2}\left(n-\frac{1}{2}\right)^{2}+\sum_{r k m \sigma} V_{r k m} d_{m \sigma}^{\dagger} c_{r k \sigma}+\text { c.c. },
\end{aligned}
$$

where $c_{r k \sigma}$ and $d_{m \sigma}$ are the Fermi operators for electrons at energies $\epsilon_{m \sigma}$ for dot electrons and $\varepsilon_{r k \sigma}$ for leads; $U$ is the Coulomb energy.

In our work two main regimes are investigated: with $n=1$ and with $n=2$ electrons on the dot. This leads to two possible states of the dot for each regime, when we take magnetic field influence $\left(\Delta_{\mathrm{Z}} \neq 0\right)$ into account and the fact that $\delta \gg k_{\mathrm{B}} T, e V$. For $n=1$ orbital $B$ is empty, and only the spin varies on the dot, while for $n=2$, orbital $A$ can be either doubly occupied and then it forms a singlet state $|S\rangle=d_{A \uparrow}^{\dagger} d_{A \downarrow}^{\dagger}|0\rangle$ and orbital $B$ is empty, or together with $B$ is singly occupied, which forms a triplet state $|T+\rangle=d_{A \uparrow}^{\dagger} d_{B \uparrow}^{\dagger}|0\rangle$. As we are in a weak coupling regime, the intra-dot spin-flip and orbital-orbital relaxation processes become relevant, and have to be taken into account to explain the details of the experimental data. 


\section{Theoretical results}

Using the second order perturbation theory $[9,10]$ we investigate cotunneling transport current for a fixed value of the bias voltage $V$ as a function of magnetic field $\Delta$. Figures 1 and 2 demonstrate the transport current at the resonance condition for various pseudo-spin asymmetries, for weak (Fig. 1), $R_{\mathrm{x}} \ll \Gamma$, and strong (Fig. 2), $R_{\mathrm{x}} \gg \Gamma$ spin-flip relaxation. We have modelled the asymmetry with two parameters: $P_{\mathrm{P}}$ and $P_{\mathrm{A}}, \Gamma_{\mathrm{L}}^{A, B \sigma}=$ $\left(1 \pm P_{\mathrm{A}}\right)\left(1 \mp P_{\mathrm{P}}\right) \Gamma$ and $\Gamma_{\mathrm{R}}^{A, B \sigma}=\left(1 \mp P_{\mathrm{A}}\right)\left(1 \mp P_{\mathrm{P}}\right) \Gamma$. Here, $P_{\mathrm{P}}$ describes asymmetry in the coupling of orbitals $A$ and $B$ to the both leads, and $P_{\mathrm{A}}$ - asymmetry in coupling opposite for both leads. For the lack of asymmetry in Fig. 1a one can observe pronounced peak at the resonance due to inelastic cotunneling and background weakly dependent on $\Delta$ due to elastic cotunneling, where in the presence of weak spin-flip relaxation the peak has a parabolic shape, Fig. 1a, and for the opposite regime a linear one, Fig. 2a. In the presence of the asymmetry

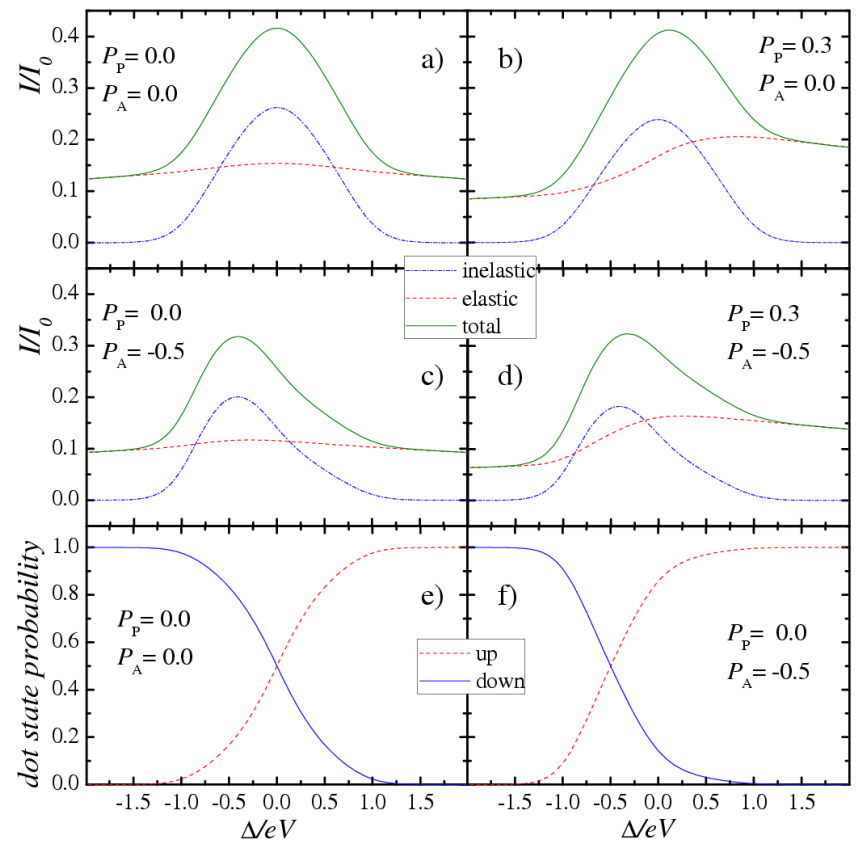

Fig. 1. Calculated cotunneling line shape as a function of the tuned magnetic field from the resonance condition for various pseudo-spin asymmetries for long (pseudo) spin-flip relaxation time. The contribution from the elastic (dashed line) and the inelastic cotunneling (dotted-dashed line) is depicted. Plots are (a) for a full symmetry $P_{\mathrm{P}}=0$ and $P_{\mathrm{A}}=0$; (b) effective parallel alignment $P_{\mathrm{P}}=0.3$; (c) effective antiparallel alignment $P_{\mathrm{A}}=-0.5 ;(\mathrm{d})$ both asymmetries are present: $P_{\mathrm{P}}=0.3$ and $P_{\mathrm{A}}=-0.5$; (e) and (f) show probabilities for spin up and down on the dot for full symmetry (e) and for $P_{\mathrm{P}}=0, P_{\mathrm{A}}=-0.5(\mathrm{f})$. Other parameters are $T / e V=0.1, \epsilon / e V=-7.8, U / e V=-26.7$, and $I_{0}=e \Gamma_{\mathrm{L}} \Gamma_{\mathrm{R}} /(2 \pi \hbar U)$.

in coupling of orbitals $A$ and $B, P_{\mathrm{P}} \neq 0$, while keeping symmetry in coupling to the both leads (Fig. $1 \mathrm{~b}$ and $2 \mathrm{~b}$ ),

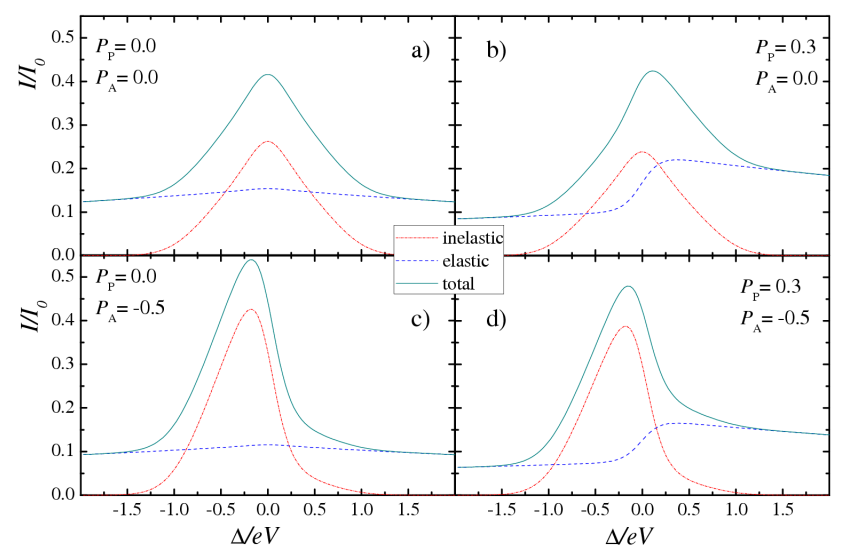

Fig. 2. Calculated cotunneling line shape as a function of the tuned magnetic field from the resonance condition for various pseudo-spin asymmetries for short (pseudo) spin-flip relaxation time. The contribution from the elastic (dashed line) and the inelastic cotunneling (dotted-dashed line) is depicted. Plots are (a) for a full symmetry $P_{\mathrm{P}}=0$ and $P_{\mathrm{A}}=0 ;(\mathrm{b})$ effective parallel alignment $P_{\mathrm{P}}=0.3$; (c) effective antiparallel alignment $P_{\mathrm{A}}=-0.5 ;(\mathrm{d})$ both asymmetries are present: $P_{\mathrm{P}}=0.3$ and $P_{\mathrm{A}}=-0.5$. Other parameters are $T / \mathrm{eV}=0.1$, $\epsilon / e V=-7.8, U / e V=-26.7$, and $I_{0}=e \Gamma_{\mathrm{L}} \Gamma_{\mathrm{R}} /(2 \pi \hbar U)$.

the inelastic cotunneling line does not change the shape, since both orbitals participate in each inelastic process. Only its amplitude is reduced by $\left(1-P_{\mathrm{P}}^{2}\right)$. For the elastic transport, there is formation of the step since, depending on the sign of $\Delta$, only one of the orbitals is strongly populated and only one of them is strongly coupled to the electrodes. It leads to enhancement of transport by $\left(1+P_{\mathrm{P}}\right)^{2}$ and reduction by $\left(1-P_{\mathrm{P}}\right)^{2}$ for opposite sides. The total line shape becomes asymmetrical and maximum is slightly shifted away from $\Delta=0$. For antisymmetric coupling of orbitals, $P_{\mathrm{A}} \neq 0$, there is symmetry in the total coupling for elastic processes, so that amplitude is only reduced by $\left(1-P_{\mathrm{A}}^{2}\right)$, and strong asymmetry in the coupling for the inelastic transport that leads to an additional spin accumulation and strong asymmetry in the inelastic line shape depending on the sign of $\Delta$ (Fig. 1c and 2c). The pronounced increase in the amplitude of the inelastic component in the presence of the strong spin-flip relaxation, $R_{\mathrm{x}} \gg \Gamma$ (Fig. 2c) proves the role of the spin accumulation that suppresses the transport in the opposite limit, $R_{\mathrm{x}} \ll \Gamma$ (the spin blockade). For strong relaxation, $R_{\mathrm{x}} \gg \Gamma$, the ratio of the transport between the positive and negative value of $\Delta$ is of order $\left[\left(1-P_{\mathrm{A}}\right) /\left(1+P_{\mathrm{A}}\right)\right]^{2}$ leading to pronounced asymmetry and shift of the total maximum away from $\Delta=0$. When both $P_{\mathrm{P}} \neq 0$ and $P_{\mathrm{A}} \neq 0$ (Fig. $1 \mathrm{~d}$ and $2 \mathrm{~d}$ ) we observe superposition of the effects discussed above, from Fig. 1b, c, and Fig. 2b, c, respectively. Figure 1e and f shows probabilities of the dot to be in spin up $\sigma=\uparrow$ or down $\sigma=\downarrow$ state for chosen setups as well (for strong spin-flip relaxation, they follow the Boltzmann distribution). The difference between Fig. 1e and f (especially 
asymmetry between positive and negative $\Delta$ ) is mainly due to the presence of the spin accumulation for $P_{\mathrm{A}} \neq 0$.

\section{Experimental results}

Our sample was comprised of an individual SWNT contacted by evaporating $\mathrm{Ti}(40 \mathrm{~nm})$ source and drain electrodes on a $\mathrm{SiO}_{2}$, and a heavily $p$-doped Si substrate was used for the gate voltage. The distance between the contacts was designed to be $300 \mathrm{~nm}$. In the majority of our devices, the whole nanotube between the contacts behaved as a single quantum dot, which showed CB oscillations with regular series at low temperatures [3, 4]. All measurements were performed in a dilution refrigerator and a magnetic field was applied perpendicularly to the tube axis.

We study in detail the line shape of the additional signal due to the cotunneling transport. Figure 3a shows the theoretical fitting of the cotunneling current taken from the dot with $n=1$ electron. The transport is modelled, using fitting parameters obtained for the sequential regime. In Fig. 3a one can distinguish inelastic components (blue lines) that exist only for $\mathrm{eV}>\Delta$ (here $\Delta=\Delta_{\mathrm{Z}}$ ) and elastic ones (red lines) weakly depending on $B$ serving as a background for the inelastic component. For a weak spin-flip relaxation, $R_{\mathrm{x}} \ll \Gamma$, theory predicts parabolic dependence of the current for $\Delta / e V \ll 1$. There is a clear discrepancy between this prediction (Fig. 3a, dashed line) and experimental results. Therefore, it is necessary to study opposite limit $R_{\mathrm{x}} \gg \Gamma$ and then theory predicts a linear dependence on $B$ which makes for a good agreement with experiment indicating that the strong spin-flip relaxation occurs in the QD.

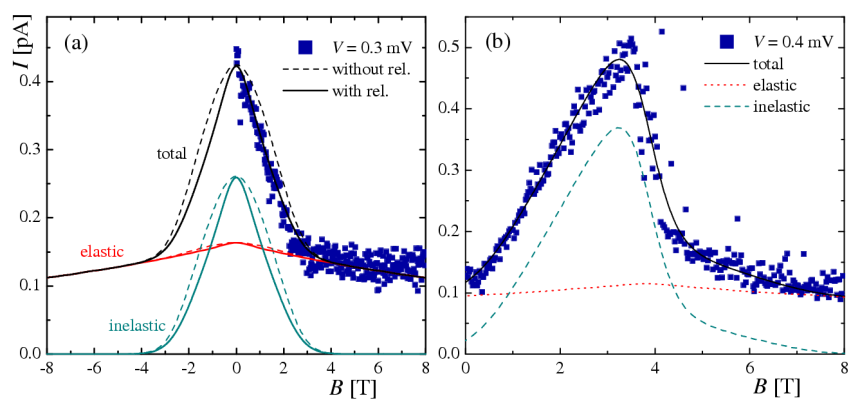

Fig. 3. (a) Cotunneling current taken from the valley with a single occupancy $(n=1)$. Solid lines are theory plots for inelastic, elastic, and total cotunneling currents for $S=1 / 2$ at the dot with strong (and the dotted for weak) relaxation, $V=0.3 \mathrm{mV}$ and (b) cotunneling current for the singlet-triplet transition at half-shell filling $(n=2)$ for $V=0.4 \mathrm{mV}$. The theory lines are plotted for the spin asymmetry $P_{\mathrm{A}}=0.45$ and $P_{\mathrm{P}}=0.0$, and with strong relaxation.

We have also investigated the cotunneling current for the singlet-triplet transition, however, a situation became more complex since the asymmetry in the coupling was possible, $P_{\mathrm{P}} \neq 0$ or $/$ and $P_{\mathrm{A}} \neq 0$. It is interesting that one can interpret the asymmetry parameters as an effective pseudo-spin polarization since both orbitals that participate in transport possess opposite spin indices. Figure $3 \mathrm{~b}$ shows experimental results and theoretical cotunneling line shape for $n=2$ electrons as a function of the tuned magnetic field, $B$. Here, the parameters $\Delta=\epsilon_{B \downarrow}-\epsilon_{A \uparrow}$ and can be also tuned by means of the magnetic field. Good agreement with theory allows for extraction of values of the asymmetry parameters fitting leads to following spin asymmetry: $P_{\mathrm{A}}=0.45$ and $P_{\mathrm{P}}=0.0$.

\section{Discussion}

The fact that the coupling of both orbitals ( $A$ and $B$ ) to the leads can be different leads to effective spin asymmetry. This situation is similar then to a dot coupled to ferromagnetic leads [10], where the spin asymmetry appears in the natural way due to spin-dependent density of states. The pseudo-spin asymmetries, $P_{\mathrm{P}}$ and $P_{\mathrm{A}}$ can be interpreted as effective spin polarization for the ferromagnetic leads with parallel and antiparallel alignment, respectively. The value of the effective spin polarization can be modified and controlled by the gate voltage by means of the electric field that can be important for the effective spin control and its manipulation at the dot.

\section{Acknowledgments}

We thank J. Barnaś, J. König, S. Maekawa, Yu.V. Nazarov, Y. Utsumi, I. Weymann, G. Schön for helpful discussions. This work was supported in part by the Grant-in-Aid for Young Scientists (B) (18710124) from the Ministry of ECSST of Japan, the Polish grant for science in years 2006-2009 as a research project, and the Center for Advance Studies at the Norwegian Academy of Science and Letters. J.M. gratefully acknowledges the hospitality of the Centre de Physique Theorique — CNRS - Luminy, Marseille, France.

\section{References}

[1] R. Saito, G. Dresselhaus, M.S. Dresselhaus, Physical Properties of Carbon Nanotubes, Imperial College Press, London 1998.

[2] D.H. Cobden, M. Bockrath, P.L. McEuen, A.G. Rinzler, R.E. Smalley, Phys. Rev. Lett. 81, 681 (1998).

[3] S. Moriyama, T. Fuse, M. Suzuki, Y. Aoyagi, K. Ishibashi, Phys. Rev. Lett. 94, 186806 (2005).

[4] S. Moriyama, T. Fuse, Y. Aoyagi, K. Ishibashi, Appl. Phys. Lett. 87, 073103 (2005).

[5] S. De Franceschi, S. Sasaki, J.M. Elzerman, W.G. van der Wiel, S. Tarucha, L.P. Kouwenhoven, Phys. Rev. Lett. 86, 878 (2001).

[6] D.M. Zumbühl, M. Marcus, M.P. Hanson, A.C. Gossard, Phys. Rev. Lett. 93, 256801 (2004).

[7] A. Kogan, S. Amasha, D. Goldhaber-Gordon, G. Granger, M.A. Kastner, Hadas Shtrikman, Phys. Rev. Lett. 93, 166602 (2004). 
[8] R. Schleser, T. Ihn, E. Ruh, K. Ensslin, M. Tews, D. Pfannkuche, D.C. Driscoll, A.C. Gossard, Phys. Rev. Lett. 94, 206805 (2005).

[9] D.V. Averin, A.A. Odintsov, Phys. Lett. A 140, 251 (1989); D.V. Averin, Yu.V. Nazarov, Phys. Rev. Lett. 65, 2446 (1990); K. Kang, B.I. Min, Phys. Rev. B 55, 15412 (1997).

[10] I. Weymann, J. Barnaś, J. König, J. Martinek, G. Schön, Phys. Rev. B 72, 113301 (2005); $\mathbf{7 2}$ 115334 (2005).

[11] S. Sasaki, S. De Franceschi, J.M. Elzerman, W.G. van der Wiel, M. Eto, S. Tarucha, L.P. Kouwenhoven, Nature 405, 764 (2000).
[12] A. Kogan, G. Granger, M.A. Kastner, D. Goldhaber-Gordon, H. Shtrikman, Phys. Rev. B 67, 113309 (2003).

[13] B. Babić, T. Kontos, C. Schönenberger, Phys. Rev. B 70, 235419 (2004).

[14] J. Paaske, A. Rosh, P. Wölfle, N. Mason, C.M. Marcus, J. Nygård, Nature Phys. 2, 460 (2006). 